Fecha de recepción: mayo 2021 Fecha de aceptación: junio 2021 Versión final: julio 2021

\section{Adaptación de la actividad proyectual del taller de diseño presencial al entorno virtual}

Eugenia Sánchez Ramos ${ }^{(1)}$, Carmen Dolores Barroso

García $^{(2)}$ y Miryam Montoya López ${ }^{(3)}$

Resumen: La educación de todos los niveles educativos tuvo un cambio radical en las instituciones educativas a nivel mundial a partir de la pandemia COVID-19, las plataformas virtuales en este sentido suplieron la clase presencial impactando de forma significativa las disciplinas de arquitectura y diseño. El presente trabajo se enfoca a la exposición vivencial de la transformación del proceso proyectual presencial en el taller de diseño al entorno virtual, así como la implementación de estrategias y aplicaciones necesarias que permiten la revisión sincrónica y asincrónica. La propuesta de un modelo de enseñanza virtual requiere en este sentido de una exploración de diversas aplicaciones que están al alcance de los estudiantes evitando la deserción y marginación.

Palabras clave: diseño - objetual - virtualidad - enseñanza - pandemia.

[Resúmenes en inglés y portugués en la página 166]

(1) Licenciada en Diseño Gráfico (Universidad de Guanajuato), Maestría en Artes Visuales (Universidad Nacional Autónoma de México), y Doctorado en Arquitectura, Diseño y Urbanismo (Universidad Autónoma del Estado de Morelos). Profesora de Tiempo Completo en la División de Ciencias Económico Administrativas de la Universidad de Guanajuato.

(2) Licenciada en Arquitectura (Universidad de Guanajuato), Maestría en Restauración y Conservación de Monumentos (Universidad de Guanajuato), y Doctorado en Arquitectura, Diseño y Urbanismo (Universidad Autónoma del Estado de Morelos). Profesora de Tiempo Completo en la División de Arquitectura, Arte y Diseño de la Universidad de Guanajuato.

(3) Licenciada en Diseño Gráfico (Universidad Vasco de Quiroga), Maestría en Administración (Universidad Vasco de Quiroga), y doctorante en el Doctorado en Artes Universidad de Guanajuato). Profesora de Tiempo Parcial en la División de Arquitectura, Arte y Diseño de la Universidad de Guanajuato. 


\section{Introducción}

El expansivo desarrollo de las Tecnologías de la Información y la Comunicación (TIC), que las sociedades han experimentado en las últimas décadas, ha marcado los modelos de educación superior, sus estructuras de gestión y sus impactos. La incorporación de las TIC en los procesos de educación superior alerta sobre nuevas formas de pensar, interpretar y hacer la educación, e impulsan la educación a distancia en línea como una modalidad socialmente aceptada, para satisfacer las necesidades educativas de amplios sectores de la población.

En este contexto recobra importancia la educación a distancia (ED). De acuerdo con Bringué y Sádaba (2009) los jóvenes nativos interactivos se forman y desarrollan en un escenario tecnológico e inestable. Sin embargo, no basta con manejar la tecnología, sino que ser competente digital es imprescindible (citado en Blanco y Cuenca, 2016, p. 104). Los estudiantes son autodidactas en el contexto del internet, es por tanto lógica la necesidad de rediseñar el proceso educativo, así como el rol del docente en el aula. Valerio y Valenzuela (2011, p. 669) difieren que los jóvenes sean nativos digitales sino informívoros quienes aprovechan las aplicaciones de las redes sociales como fuente de información.

En la actualidad Blanco y Cuenca (2016) señalan que es una realidad que las tecnologías digitales han influido en la manera de aprender, así como en la enseñanza de los docentes. Siguiendo con los autores, resulta importante retomar la Teoría del Conectivismo (Siemens, 2006), la cual estudia el aprendizaje en red misma que se lleva a cabo por medio de las conexiones y que integra principios como el caos, la complejidad y la autoorganización. Es, por tanto, un aprendizaje no individual y se orienta a la co-creación del conocimiento gracias a la interacción de las personas, en este sentido es más importante saber dónde y saber quién, que saber qué y cómo.

Benavides, Villacís, \& Ramos (2017) establecen que:

Un Entorno Virtual de Aprendizaje (EVA) tiene un sin número de actividades o herramientas, las cuales deben ser utilizadas y aprovechadas adecuadamente por los docentes y estudiantes en los centros educativos, especialmente de nivel medio y superior, con el objetivo de crear o generar nuevo conocimiento (p. 47).

En los EVA, existen tres niveles de conocimientos con diferentes grado de profundidad que interactúan constantemente (Benavides, Villacís, \& Ramos, 2017, p. 48) y son: 


\begin{tabular}{|l|c|l|}
\hline Nivel 1 & $\begin{array}{c}\text { Modelo Difuso } \\
\text { (MODI) }\end{array}$ & $\begin{array}{l}\text { Transmisión de contenidos por parte del profesor, no } \\
\text { favorece la acumulación-adquisición de conocimiento, y } \\
\text { no desarrolla nuevos saberes. }\end{array}$ \\
\hline Nivel 2 & $\begin{array}{c}\text { Modelo Orientado a } \\
\text { la Enseñanza } \\
\text { (MOEN) }\end{array}$ & $\begin{array}{l}\text { Favorece procesos de acumulación y adquisición de } \\
\text { saberes, así como la acumulación-adquisición, pero no } \\
\text { desarrolla nuevos saberes. Informa sobre el uso del } \\
\text { espacio virtual e información, fomenta la adquisición } \\
\text { competencial. }\end{array}$ \\
\hline Nivel 3 & $\begin{array}{c}\text { Modelo Orientado al } \\
\text { Aprendizaje } \\
\text { (MOAP) }\end{array}$ & $\begin{array}{l}\text { Desarrolla e inventa nuevos saberes, así como nuevas } \\
\text { formas de trabajar el contenido, favorece la acumulación- } \\
\text { adquisición y sín desarrolla nuevos saberes; se centra } \\
\text { en el apoyo a la docencia (informar, consultar } \\
\text { documentos, etc.) y al aprendizaje (participativo, } \\
\text { dinámico, etc.); promociona la comunicación e } \\
\text { interacción docente-estudiante y estudiante - estudiante }\end{array}$ \\
\hline
\end{tabular}

Tabla1. Niveles de conocimiento en entornos virtuales.

Fuente: Elaboración en base a Benavides, Villacís, \& Ramos (2017).

En cuanto a la enseñanza del taller de diseño en la virtualidad, que es el caso que ocupa el presente trabajo, Dalle (2019) menciona que

El grado de exposición del objeto en el entorno virtual dista del presencial. No es igual el acercamiento a la cosa diseñada en ambos ámbitos. En el entorno virtual el sentido del tacto y del olfato queda reducido, la cosa no se puede manipular, tocar y prima la imaginación. Si bien tenemos un acercamiento desde el sentido de la visualidad, estamos ante réplicas de la simulación entendiendo que aún lo entregado al final del ejercicio es la simulación de productos y objetos que podrían intervenir en el seno social. (p.78)

Es decir, las actividades en la virtualidad se basan en las actividades presenciales de taller, sin embargo, la hipermedia permite mejorar la experiencia visual a través del video, imagen, esquemas, simulación $3 \mathrm{~d}$, etc. al respecto brevedad e inmediatez son parte del universo convergente mediático (Igarza, 2008). La actividad proyectual, por tanto, se enriquece ya que los dispositivos y plataformas permiten documentar el proceso de diseño. En este sentido, el docente debe buscar aplicaciones que permitan la revisión de la propuesta de forma sincrónica lo que promueve la generación de nuevas metodologías enfocada a la reorganización de elementos de diseño y a los procesos de ejecución.

En resumen, los Entornos Virtuales de Aprendizaje (EVA) exigen la evolución de la enseñanza a distancia convencional que generalmente se orienta la administración de cursos teóricos, esto debido a que no es suficiente la utilización de plataformas estandarizados para el desarrollo de la actividad proyectual por lo que es imperante complementar el 
proceso de diseño mediante otras herramientas, dispositivos y aplicaciones que mejoran y optimizan la propuesta.

\section{Desarrollo}

La experiencia se llevó a cabo durante la contingencia sanitaria COVID-19 la cuál impidió el regreso a clases presenciales desde el 17 de marzo de 2020 implementándose la educación virtual hasta este momento. El objetivo primordial es rediseñar la UDA Taller de Diseño Básico impartida en la Lic. en Arquitectura de la Universidad de Guanajuato utilizando la plataforma TEAMS. Para el desarrollo del proceso se eligió la metodología de caso, lo cual establece una investigación cualitativa inductiva para realizar un diagnóstico previo de las competencias de los estudiantes en torno a la utilización de la herramienta, así como de los conocimientos previos teóricos.

Derivado del contexto, la perspectiva de aprendizaje que se planteó para esta experiencia fue el B-Learning ${ }^{l}$, ya que de acuerdo a Llovero y Cabero (2008) combina la eficacia y la eficiencia de la clase presencial con la flexibilidad del eLearning, teniendo como recursos de aprendizaje y vías de comunicación entre tutor-estudiante y estudiante-estudiante incidiendo en una comunicación más interactiva.

\section{Metodología}

1. Definición de las dimensiones de la competencia digital (CD). El rediseño de la UDA se sustenta en las Dimensiones de la competencia digital las cuales son (ver fig. 1): 
Información: identificar, localizar, recuperar, almacenar, organizary analizar la información digital, evaluando su finalidad y relevancia.

Comunicación: compartir recursos a través de herramientas en línea, interactuar y participar en comunidades y redes; conciencia intercultural.

Creación de contenido, integrar y reelaborar conocimientos y contenidos previos, contenidos multimedia y programación informática, saber aplicar los derechos de propiedad intelectual y las licencias de uso.

Seguridad: protección personal, protección de datos, protección de la identidad digital, uso de seguridad, uso seguro y sostenible.

Resolución de problemas: identificar necesidades y recursos digitales, seleccionar la herramienta digital, resolver problemas conceptuales y técnicos, uso creativo de la tecnología, actualizar la competencia propia y la de otros.

Figura 1. Dimensiones de la competencia digital. Fuente: Elaboración propia basada en Blanco y Cuenca (2016)

2. Diagnóstico mediante la aplicación de un cuestionario que se basó en dos categorías: manejo de plataformas virtuales en específico TEAMS, acceso a la conectividad, y conocimientos previos revisados en las sesiones presenciales. La aplicación del cuestionario al grupo permitió identificar que de los 100 alumnos registrados el 3\% utilizaban el lápiz electrónico; el 41.2\% tenía problemas de conectividad para comunicación sincrónica, $10 \%$ conocía y aplicaba la aplicación de block de notas. En cuanto a la revisión de conocimientos previos la totalidad no presentó rezago. Se constata que los estudiantes procesan información de las redes sociales (ver Fig. 2, 3 y 4). 
4. Dispositivo con el cual te conectaste hoy

Más detalles

$\begin{array}{ll}\text { Computadora de escritorio } & 2 \\ \text { Laptop } & 49 \\ \text { Celular } & 25 \\ \text { Tableta } & 0\end{array}$

Figura 2. Encuesta de diagnóstico. Fuente: Elaboración propia (2020)

Preguntas

\section{Dispositivo del que te conectaste hoy}

Más detalles

$$
\begin{aligned}
& \text { Laptop } \\
& \text { celular }
\end{aligned}
$$

Otras

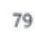

0

32

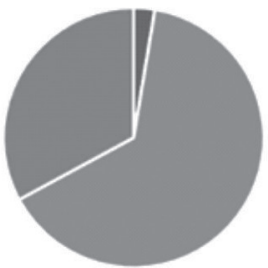

5


3. Rediseño de contenido y actividades prácticas del entorno presencial al virtual. Teniendo como base la definición Informívoro de George Miller (Valerio y Valenzuela, 2011) se siguió el modelo que se describe en la Figura 5:

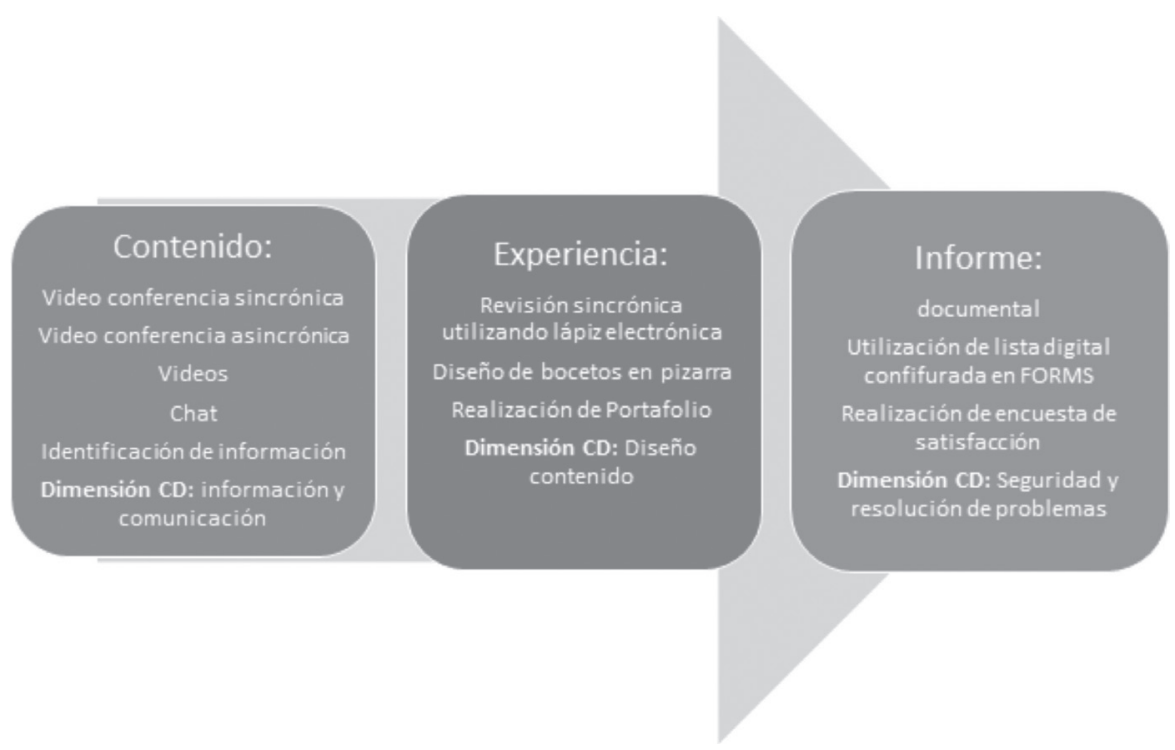

Figura 5. Estructuración de UDA VIRTUAL. Fuente: Elaboración propia (2020)

Como se puede observar, dentro de estas tres fases sistémicas se relacionaron las dimensiones de la $\mathrm{CD}$, estableciendo la comunicación sincrónica y asincrónica que permite la plataforma TEAMS al grabar las sesiones para posterior consulta de los estudiantes que tuvieran dificultades con la conexión.

En cuanto a la experiencia, el objetivo de la UDA es la realización de ejercicios utilizando aplicaciones como power point en donde la utilización del lápiz electrónico es posible evitando el envío de trabajos en formato PDF, y la pizarra White board para la revisión de la organización de los elementos del diseño. En este apartado se utilizó el block de notas de clase como bitácora del proceso de revisión y configuración promoviendo la interacción de los miembros en la discusión y edición de la información.

Finalmente, la tercera fase se optó por la edición de un Portafolio digital como evidencia el trabajo realizado en el semestre, esto sugiere la presentación del proceso de preconfiguración, configuración y modelación de los ejercicios establecidos en el programa. 
4. Evaluación. La plataforma permite la entrega de cada tema garantizando que los equipos cumplan con las fechas establecidas, así como de la rúbrica de cada tarea solicitada (ver Fig. 6 y 7). La asistencia a clase, establecida en la normativa de la Ley Orgánica de la Universidad de Guanajuato se estableció mediante una lista electrónica en FORMS el cual genera un archivo de Word permite tener control de actividades, reacciones y conectividad.

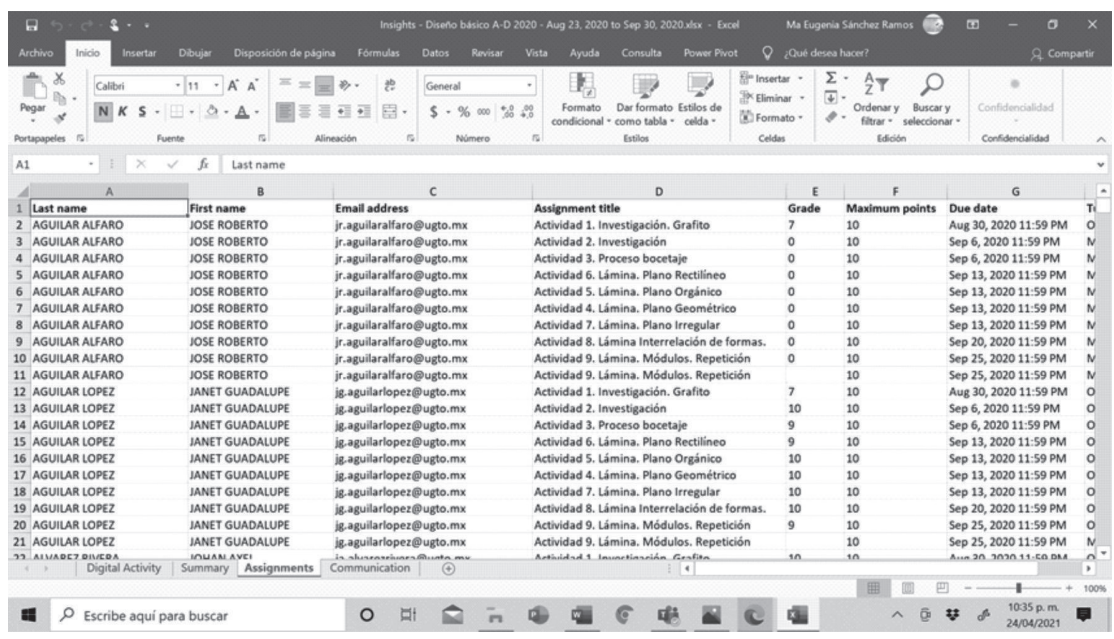

Figura 6. Control de actividades y lista de asistencia. Fuente: Elaboración propia (2020)

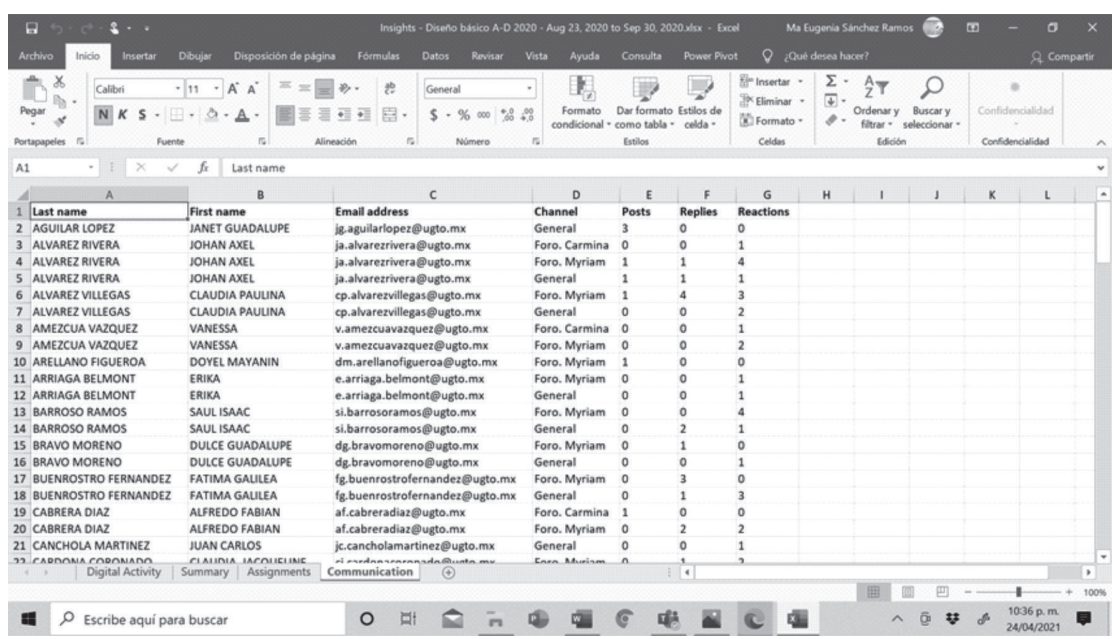

Figura 7. Control de respuesta y participaciones. Fuente: Elaboración propia (2020) 
Para la retroalimentación de la labor docente se diseñó un formulario en línea para conocer la opinión de los estudiantes en torno al rediseño de la UDA virtual. Los datos recopilados señalan que los jóvenes tienen una gran destreza para las redes sociales, pero tanto el docente como el alumno se encuentra en desarrollo de las competencias que le permitan manipular de forma óptima las plataformas educativas.

\section{Conclusión}

Si bien la comunidad estudiantil ha crecido en un ámbito digital, el profesorado está obligado a actualizarse en el proceso de enseñanza digital además de desarrollar las competencias necesarias que exige este medio. Lo que se había discutido anteriormente en foros y congresos académicos es hoy una realidad para la cual es necesario no únicamente ser capaz de usar tecnologías y plataformas educativas digitales sino desarrollar una visión crítica, responsable en la selección y discriminación de la información, y en la construcción del conocimiento propio.

Es momento de discutir la idea preconcebida de que las redes sociales no se vinculan con el aprendizaje y son una distracción, ya que representan el medio de interacción y comunicación entre el alumnado; se requiere por tanto de impulsar las competencias informáticas que les permitan discernir la información y construir conocimiento.

\section{Notas:}

1. El término "blended learning", se puede traducir al castellano como Aprendizaje Mezclado, sigue una tendencia con una marcada raíz procedente del campo de la psicología escolar en la que destaca el término "aprendizaje" como contrapuesto al de "enseñanza" (Bartolomé, 2004).

\section{Listado de Referencia Bibliográfica}

Bartolomé, A. (2004). Blended Learning, Conceptos Básicos. Píxel-Bit Revista de Medios y Educación, 23, 7-20. Disponible en: https://www.researchgate.net/profile/Julio_Gonzalez_Marino/publication/27591806_B-Learning_utilizando_software_libre_una_alternativa_viable_en_Educacion_Superior/links/0deec5304e295de331000000.pdf

Benavides-Lara, R., Villacís-Lizano, M., \& Ramos-Paredes, J. J. (2017). El Entorno Virtual de Aprendizaje (EVA) en la generación de conocimiento de estudiantes universitarios. CienciAmérica, 6(1), 57-63.Blanco, A. V., \& Amigo, J. C. (2016). El rol del docente en la era digital. Revista interuniversitaria de formación del profesorado, 30(2), 103-114. 
Bringué, X., \& Sádaba-Chalezquer, C. (2010). Niños y adolescentes españoles ante las pantallas: rasgos configuradores de una generación interactiva. Disponible en https:// dadun.unav.edu/handle/10171/18443

Dalle, L. (2019). Taller-mediante. Reflexiones críticas sobre una experiencia de amplificación del taller de diseño al medio virtual/digital. Cuaderno 84, 69.

Igarza, R (2008). Nuevos medios. Estrategias de convergencia. Buenos Aires: La Crujia. Capítulo N..$^{\circ}$ : "La convergencia de medios".

Llorente Cejudo, M. D. C., \& Cabero Almenara, J. (2008). Del e-learning al Blended Learning: nuevas acciones educativas. Quaderns Digitals, 51.

Siemens, G. (2006). Knowing knowledge. Lulu. com.

Valerio, G., \& Valenzuela, R. (2011). Redes sociales y estudiantes universitarios: del nativo digital al informívoro saludable. El profesional de la información, 20(6), 667-670.

\begin{abstract}
Education at all educational levels had a radical change in educational institutions worldwide from the COVID-19 pandemic, virtual platforms in this sense supplied the face-to-face class, significantly impacting the disciplines of architecture and design. This work focuses on the experiential exposition of the transformation of the face-to-face project process in the design workshop to the virtual environment, as well as the implementation of strategies and necessary applications that allow synchronous and asynchronous review. In this sense, the proposal of a virtual teaching model requires an exploration of various applications that are available to students, avoiding desertion and marginalization.
\end{abstract}

Keywords: design - object - virtuality - teaching - pandemic.

Resumo: A educação em todos os níveis educacionais teve uma mudança radical nas instituições de ensino em todo o mundo a partir da pandemia COVID-19, plataformas virtuais neste sentido abasteciam as aulas presenciais, impactando significativamente as disciplinas de arquitetura e design. Este trabalho tem como foco a exposição experiencial da transformação do processo de projeto presencial na oficina de design para o ambiente virtual, bem como a implementação de estratégias e aplicações necessárias que permitam a revisão síncrona e assíncrona. Nesse sentido, a proposta de um modelo de ensino virtual requer a exploração dos diversos aplicativos que estão à disposição dos alunos, evitando a deserção e a marginalização.

Palavras chave: design - objeto - virtualidade - ensino - pandemia.

[Las traducciones de los abstracts fueron supervisadas por el autor de cada artículo] 